\title{
Primary Leiomyosarcoma of the Breast with Unusual Metastasis
}

\author{
Malek Bouhani, ${ }^{1}$ (1) Saida Sakhri, ${ }^{1}$ (1) Olfa Jaidane,, (1) Salma Kammoun, ${ }^{2}$ \\ (D) Riadh Chargui, ${ }^{1}$ (D) Khaled Rahal ${ }^{1}$ \\ 'Department of Oncologic Surgery, Salah Azaiz Institute, Tunis, Tunisia \\ ${ }^{2}$ Department of Anatomopatholgy, Salah Azaiz Institute, Tunis, Tunisia
}

\section{ABSTRACT}

Primary leiomyosarcomas of the breast are extremely rare tumors, with unpredictable biological behavior. The main treatment consists of wide resection or mastectomy. Local recurrence is late; the distant metastasis spreads via the hematogenous route to lungs and liver; however, bone metastasis is rarely reported. Here, we present a case of primary leiomyosarcoma of the breast, diagnosed in the postmenopausal female. She underwent a wide resection with free margins. The patient rapidly, presented an unusual aggressive behavior of leiomyosarcoma, with a local recurrence in axillary lymph node and an exceptional distant metastasis in bones.

Keywords: Breast, leiomyosarcoma, neoplasm metastasis.

\section{INTRODUCTION}

Please cite this article as: Bouhani M, Sakhri S, Jaidane O, Kammoun S, Chargui R, Rahal K. Primary Leiomyosarcoma of the Breast with Unusual Metastasis. Anatol J Family Med 2019;2(3):134-136.

Address for correspondence: Dr. Malek Bouhani. Department Of Oncologic Surgery, Salah Azaiz Institute, Tunis, Tunisia

Phone: +21623604048

E-mail:

bouhani_malek@hotmail.fr

Received Date: 18.03.2019 Accepted Date: 02.05.2019 Published online: 12.11.2019

(C) Copyright 2019 by Anatolian Journal of Family Medicine Available online at www.anatoljfm.org OPEN ACCESS

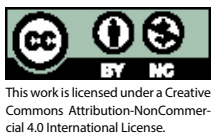

Primary leiomyosarcoma (LMS) of the breast is an extremely rare nonepithelial malignancy that occurs in the smooth muscles. LMS accounts for less than $1 \%$ of all breast tumors, and $5-10 \%$ of breast sarcomas, ${ }^{[1,2]}$ less than 50 cases reported in the English literature ${ }^{[3]}$. The diagnosis of LMS preoperatively is challenging. Owing to the lack of literature, the biological behavior in breast LMS stills uncertain.

\section{CASE REPORT}

A 50-year old postmenopausal female consulted for a right breast mass, which she noticed the lump for the past one month. There was no family history of breast or ovarian cancer. The local examination found a large, renitent, painful and mobile breast mass measuring $15 \mathrm{~cm}$ of the major axis and involving all the four quadrants. There were no skin changes. The contralateral breast was unremarkable. Routine laboratory investigations were within normal limits. Mammography showed an opacity occupying the entire breast (Fig. 1), which corresponded to ultrasound scanning that indicated a heterogeneous cystic mass (Fig. 1). An ultrasound-guided (USG) breast biopsy from the solid component was performed, but it was not contributive. Due to the large size of the cyst and the failure of the USG, we achieved a fine needle aspiration that obtained $400 \mathrm{ml}$ of citrine yellow liquid. The cytological analysis was inconclusive.

The patient underwent a lumpectomy of the mass that was accidentally fissured. The histological findings showed a sarcoma without typing. The staging workup did not show any metastatic lesions. The patient underwent mastectomy with axillary lymph node sampling. 
The microscopic pathological examination of the mastectomy specimen showed pleomorphic spindle cells with frequent mitoses, necrosis and atypical nuclei (Fig. 2). The immunohistochemical study showed that the tumor expresses actin; it was negative for desmin, PS100, and cytokeratin (Fig. 2). On the basis of the morphological findings, the tumor is reminiscent of the appearance of LMS. The axillary sampling was free of disease and the clearance of surgical margins was $1 \mathrm{~cm}$.

In the multi-disciplinary committee, despite the free surgical margins, we indicated adjuvant radiotherapy due to the large size of the tumor, as treatment guidelines of non-breast soft tissue sarcomas. The patient was lost of view without adjuvant radiotherapy. Nine months later, she returned with an axillary mass that was infected, ulcerated and fixed. The biopsy confirmed the recurrence. The computed tomography scan showed bone lysis of the fifth costal arch, homolateral pleural effusion and secondary pulmonary localizations. Chemotherapy was indicated but rejected due to the changes of the patient's general condition.
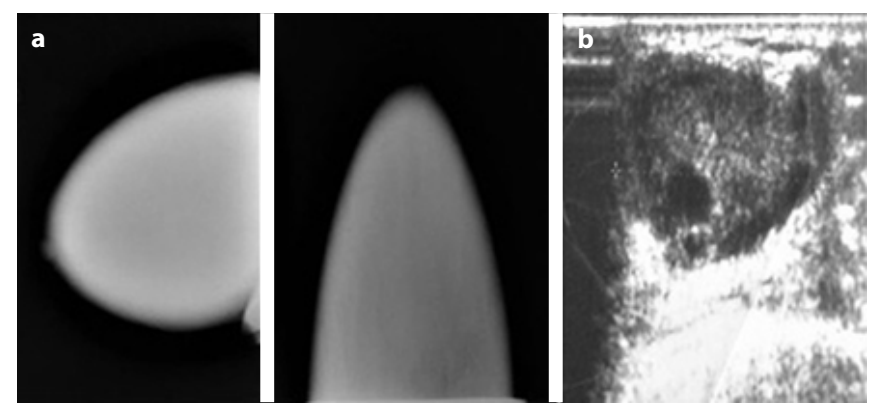

Figure 1. (a) Mammography: opacity occupying the entire breast. (b) Ultrasound: heterogeneous cystic mass.
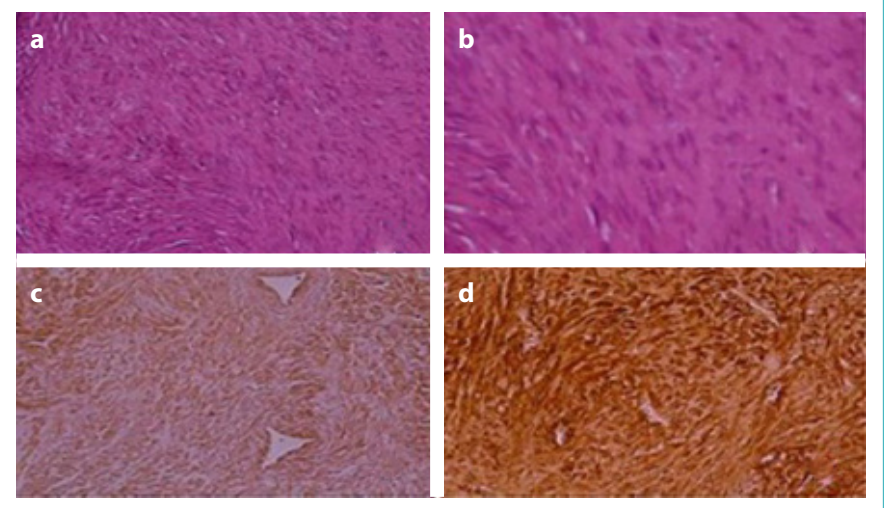

Figure 2. (a, b) Progressive increasing magnification of histology (HES 10 , and 40 ) showing a tumor composed of hyperchromatic spindle cells with frequent atypical nuclei. (c, d) Immunochemistry positivity for smooth muscle actin and h-caldesmon.

\section{DISCUSSION}

LMS is an uncommon breast tumor. The origins of LMS is still debated. Some authors suggest that LMS arises from the muscular blood vessels or from the smooth muscle of the nipple ${ }^{[3]} \mathrm{LMS}$ is generally diagnosed on a post-menopausal female with a median age of 56 years. ${ }^{[2]}$

The clinic presentation simulates a benign lesion. It is usually presented as a well-circumscribed mass ${ }^{[2]}$ and characterized by a large size with an average tumor size of $4.7 \mathrm{~cm}$ ${ }^{[4]}$. LMS rarely causes the axillary lymph node ${ }^{[3]}$ Radiological findings are nonspecific. mammography and ultrasound are mostly mistaken as a malignant lesion..$^{[2]}$

Definitive diagnosis is confirmed by histological examination. LMS is characterized by spindle cell bundles with further grading via the degree of atypia, mitotic activity, cellularity, and nuclear pleomorphism. In the immunohistochemical stains, LMS expresses desmin, vimentin, and muscle-specific actin, but LMS is negative for cytokeratin, myoglobin, and S-100. ${ }^{[1-3]}$

Due to the paucity of cases treated in the literature, treatment guidelines have been extrapolated from studies of non-breast soft tissue sarcomas. The main treatment is surgical excision with free surgical margins or mastectomy. However, we should note that the authors recommended negative margins more than two centimeters in the case of conservative surgery. ${ }^{[1]}$ Due to the rarity of lymph node metastasis, authors do not recommend lymph node dissection; meanwhile, sentinel lymph node dissection could be an alternative. ${ }^{[2,5]}$ Radiotherapy is recommended after local excision. Chemotherapy is indicated for the metastatic stage; however, the benefits are not yet confirmed. ${ }^{[2]} \mathrm{An}$ thracyclines and ifosfamide are currently used..$^{[1]}$

LMS tends to have a local recurrence if the resection margins are inadequate. However, when negative margins are achieved, ${ }^{[1]}$ this recurrence may ariseeven after 15-20 years, unlike our case that presented recurrence after nine months. ${ }^{[2]}$

Distant metastasis that spread via the hematogenous route was observed in $25 \%$ of patients; it is located in the lungs, liver, and bones, ${ }^{[2,3]}$ and also in our case as we reported.

The prognosis is better than the other breast sarcomas. Prognostic factors still not well established due to the limited number of cases. Five-year survival rates are $63 \%$, $36 \%$, and $14 \%$ for localized, regional, and distant diseases respectively, that is lead to a long term of follow up. ${ }^{[1,2]}$

\section{CONCLUSION}

In conclusion, we present a rare case of LMS, an unusual clinical behavior. 


\section{Disclosures}

Informed Consent: Written informed consent was obtained from the parents of the patient for the publication of the case report and the accompanying images.

Conflict of Interest: The authors declared no conflict of interest. Peer-review: Externally peer-reviewed.

Financial Disclosure: There is no funding in this article.

Authorship Contributions: Concept - R.C.; Design - S.S..; Supervision - M.B.; Fundings - K.R.; Materials - S.K.; Data collection \&/or processing - M.B.; Analysis and/or interpretation - M.B.; Literature search - O.J.; Writing - S.S.; Critical Review - O.J.

\section{REFERENCES}

1. Amaadour L, Benbrahim Z, Moumna K, Boudahna L, Amarti A, Arifi S, et al. Primary breast leiomyosarcoma. Case Rep Oncol Med 2013;2013:732730. [CrossRef]

2. Karabulut Z, Akkaya H, Moray G. Primary Leiomyosarcoma of the Breast: A Case Report. J Breast Cancer 2012;15(1):124-7.

3. Singh G, Sharma D, Goyal S. Primary leiomyosarcoma of breast presenting with metastasis: An atypical presentation with dismal prognosis. Indian J Med Paediatr Oncol 2017;38(4):535-7.

4. Oktay Y, Fikret A. Leiomyosarcoma of the breast. J Surg Case Rep 2011;2011(7):1. [CrossRef]

5. Székely E, Madaras L, Kulka J, Járay B, Nagy L. Leiomyosarcoma of the female breast. Pathol Oncol Res 2001;7(2):151-3. [CrossRef] 